\title{
Article
}

\section{On the Discrete Weibull Marshall-Olkin Family of Distributions: Properties, Characterizations, and Applications}

\author{
Jiju Gillariose ${ }^{1}\left(\mathbb{0}\right.$, Oluwafemi Samson Balogun ${ }^{2, *}\left(\mathbb{D}\right.$, Ehab M. Almetwally ${ }^{3}$, Rehan Ahmad Khan Sherwani ${ }^{4}$, \\ Farrukh Jamal ${ }^{5, *}$ (D) and Joshin Joseph ${ }^{6}$ (i)
}

1 Department of Statistics, CHRIST (Deemed to Be University), Bangalore 560029, India; jijugillariose@yahoo.com

2 School of Computing, University of Eastern Finland, 70211 Kuopio, Finland

3 Department of Statistics, Delta University of Science and Technology, Belkas 35631, Egypt; ehabxp_2009@hotmail.com

4 College of Statistical and Actuarial Sciences, University of the Punjab, Lahore 54000, Pakistan; rehan.stat@pu.edu.pk

5 Department of Statistics, The Islamia University of Bahawalpur, Bahawalpur 63100, Pakistan

6 Department of Finance, KMML a Company under Government of Kerala, Sankaramangalam, Kollam 691583, India; joshinpariyath@gmail.com

* Correspondence: samson.balogun@uef.fi (O.S.B.); drfarrukh1982@gmail.com (F.J.)

check for updates

Citation: Gillariose, J.; Balogun, O.S.; Almetwally, E.M.; Khan Sherwani, R.A.; Jamal, F.; Joseph, J. On the Discrete Weibull Marshall-Olkin Family of Distributions: Properties, Characterizations, and Applications. Axioms 2021, 10, 287. https:// doi.org/10.3390/axioms10040287

Academic Editor: Hari Mohan Srivastava

Received: 16 September 2021

Accepted: 28 October 2021

Published: 30 October 2021

Publisher's Note: MDPI stays neutral with regard to jurisdictional claims in published maps and institutional affiliations.

Copyright: (C) 2021 by the authors. Licensee MDPI, Basel, Switzerland. This article is an open access article distributed under the terms and conditions of the Creative Commons Attribution (CC BY) license (https:/ / creativecommons.org/licenses/by/ $4.0 /)$.

\begin{abstract}
In this article, we introduce a new flexible discrete family of distributions, which accommodates wide collection of monotone failure rates. A sub-model of geometric distribution or a discrete generalization of the exponential model is proposed as a special case of the derived family. Besides, we point out a comprehensive record of some of its mathematical properties. Two distinct estimation methods for parameters estimation and two different methods for constructing confidence intervals are explored for the proposed distribution. In addition, three extensive Monte Carlo simulations studies are conducted to assess the advantages between estimation methods. Finally, the utility of the new model is embellished by dint of two real datasets.
\end{abstract}

Keywords: confidence interval; discrete distributions; generalized family; Marshall-Olkin family; estimation; Weibull distribution

MSC: 60E05; 62E15; 62F10

\section{Introduction}

The Marshall-Olkin family of distributions was introduced by [1]. This is an interesting endeavour for associating additional parameter to an existing baseline. The resulting new probability distributions provide more flexibility to model various kinds of data. The early studies of the this scheme by [2-8], etc. offered its features. For a comprehensive discussion, one can refer a write-up by [9]. Keeping in view its applications, researchers focused on generalizations and extensions of the Marshall-Olkinn family of distribution. For example, exponentiated Marshall-Olkin extended family [10], Harris extended family [11], truncated negative-binomial generalized Marshall-Olkin family [12], beta Marshall-Olkin family [13], Kumaraswamy Marshall-Olkin family [14], truncated discrete Mittag-Leffler family [15], Marshall-Olkin generalized family [16], truncated discrete Linnik family [17], etc.

Recently, ref. [18] proposed a new family of distributions called the Weibull MarshallOlkin generalized family. Its cumulative distribution function (cdf) with arbitrary baseline $F(x ; \eta)$, is given by

$$
G(x ; \alpha, b, \eta)=1-\exp \left(-\left\{-\log \left[\frac{\alpha \bar{F}(x, \eta)}{1-\bar{\alpha} \bar{F}(x, \eta)}\right]\right\}^{b}\right) \quad x>0, b>0, \alpha>0
$$


and $\bar{\alpha}=1-\alpha$. This family is derived based on T-X family introduced by [19].

In many practical situations, discretizing statistical models from existing continuous distributions brings a crucial role. Consequently, some discrete distributions derived based on popular continuous models, for example, see, refs. [20-25], etc. Furthermore, several discrete distributions appeared in the literature by using continuous Marshall-Olkin distributions [26-28].

The most prominent motivating factor that pushed us towards the introduction of this new discrete distribution is the that while comparing with the volume of literature in continuous case, only limited research works have been inscribed about the discrete version of continuous family of distributions. The properties of the proposed model itself is another source of motivation. That is, the proposed new discrete model has positively skewed, decreasing and symmetric probability mass function (pmf). Moreover, the new distribution has decreasing, increasing, unimodal and bathtub shaped hazard rate functions (hrf). In addition to this, we offered a comparison of the estimation methods and real-life data application to explain how well the proposed model give consistently better fit than the other well-known discrete distributions.

The rest of the paper is outlined as follows: In Section 2, we propose a new discrete family of distributions and a special case of the derived family together with its probabilistic properties. Two distinct estimation procedures for parameters estimation are illustrated in Section 3. In Section 4, we give demonstration of the two different methods for constructing confidence intervals for the proposed distribution. Section 5 inculcates the Monte Carlo simulations study. Applications to the two real data sets to illustrate the performance of the new family are explained in Section 6 . Section 7 offers some concluding remarks.

\section{Discrete Weibull Marshall-Olkin Exponential Distribution}

In this section, we propose a new discrete family of distributions namely discrete Weibull Marshall-Olkin family of distributions. Different methods and constructions of discrete analogs of continuous distributions are given in [29]. If the underlying continuous life time $X$ has the survival function $\bar{G}(x)=P(X>x)$, the pmf of the discrete random variable associated with that continuous distribution can be written as

$$
P(X=x)=\bar{G}(x)-\bar{G}(x+1) ; x=0,1,2, . .
$$

The new family is generated by discretizing the continuous survival in Equation (1) using Equation (2), we obtain new family of discrete distribution with the pmf $g(x)$. The pmf can be written as

$g(x)=\exp \left(-\left\{-\log \left[\frac{\alpha \bar{F}(x)}{1-\bar{\alpha} \bar{F}(x)}\right]\right\}^{b}\right)-\exp \left(-\left\{-\log \left[\frac{\alpha \bar{F}(x+1)}{1-\bar{\alpha} \bar{F}(x+1)}\right]\right\}^{b}\right) ; \quad x=0,1,2 \ldots$

The survival function of the discrete random variable having the pmf (3) is given by

$$
\bar{G}(x)=\exp \left(-\left\{-\log \left[\frac{\alpha \bar{F}(x+1)}{1-\bar{\alpha} \bar{F}(x+1)}\right]\right\}^{b}\right) ; \quad x=0,1,2 \ldots
$$

We study a special case of this family, namely, discrete Weibull Marshall-Olkin exponential (DWMOE) distribution. The exponential distribution was chosen because it is the most tractable model. In practice for modeling and analyzing of random phenomenons, other distributions could be used.

Let the parent distribution be exponential with parameter $\lambda$ and survival function $\bar{F}(x)=e^{-\lambda x}$. We set $\rho=e^{-\lambda}$ and $0<\rho<1$. Then, the pmf of the new model using (3) is given by

$$
g(x)=\exp \left(-\left\{-\log \left[\frac{\alpha \rho^{x}}{1-\bar{\alpha} \rho^{x}}\right]\right\}^{b}\right)-\exp \left(-\left\{-\log \left[\frac{\alpha \rho^{(x+1)}}{1-\bar{\alpha} \rho^{(x+1)}}\right]\right\}^{b}\right) ; \quad x=0,1,2 \ldots
$$


We called this new distribution the DWMOE distribution with parameters $\alpha, b$ and $\rho$. Note that, when $b=1$, the distribution with pmf (5) reduces to discrete MarshallOlkin distributions, when $b=1$ and $\alpha=1$, (5) becomes geometric distribution. The corresponding cumulative distribution function (cdf) corresponding to (5) can be written as

$$
\bar{G}(x)=\exp \left(-\left\{-\log \left[\frac{\alpha \rho^{(x+1)}}{1-\bar{\alpha} \rho^{(x+1)}}\right]\right\}^{b}\right) ; \quad x=0,1,2 \ldots
$$

The corresponding hrf is given by

$$
h(x)=\frac{\exp \left(-\left\{-\log \left[\frac{\alpha \rho^{x}}{1-\bar{\alpha} \rho^{x}}\right]\right\}^{b}\right)-\exp \left(-\left\{-\log \left[\frac{\alpha \rho^{(x+1)}}{1-\bar{\alpha} \rho(x+1)}\right]\right\}^{b}\right)}{\exp \left(-\left\{-\log \left[\frac{\alpha \rho^{(x+1)}}{1-\bar{\alpha} \rho^{(x+1)}}\right]\right\}^{b}\right)}
$$

Figure 1 shows the plots of the pmf of the DWMOE distribution for various values of $\alpha, b$ and $\rho$. The pmf can be decreasing and upside-down bathtub shaped. Furthermore, Figure 2 illustrates some of the possible shapes of the hrf of DWMOE distribution for selected values of the parameters. From figures, we can see that the hrf can be increasing, decreasing, bathtub, and upside-down bathtub shaped. Therefore, the DWMOE distribution is very efficient for modeling various data sets.
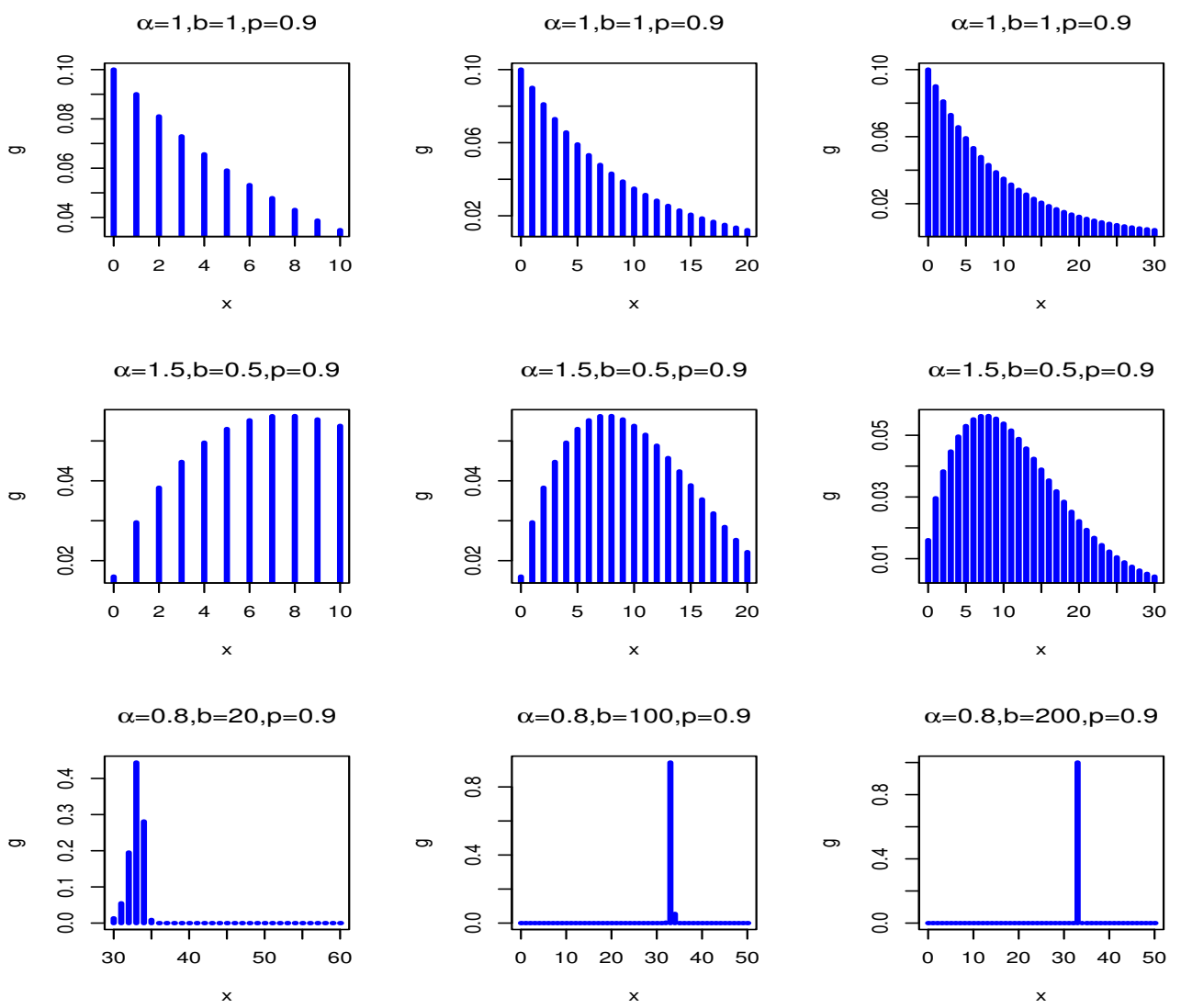

Figure 1. pmfs of the DWMOE $(\alpha, b, \rho)$ distribution for some parameter values. 

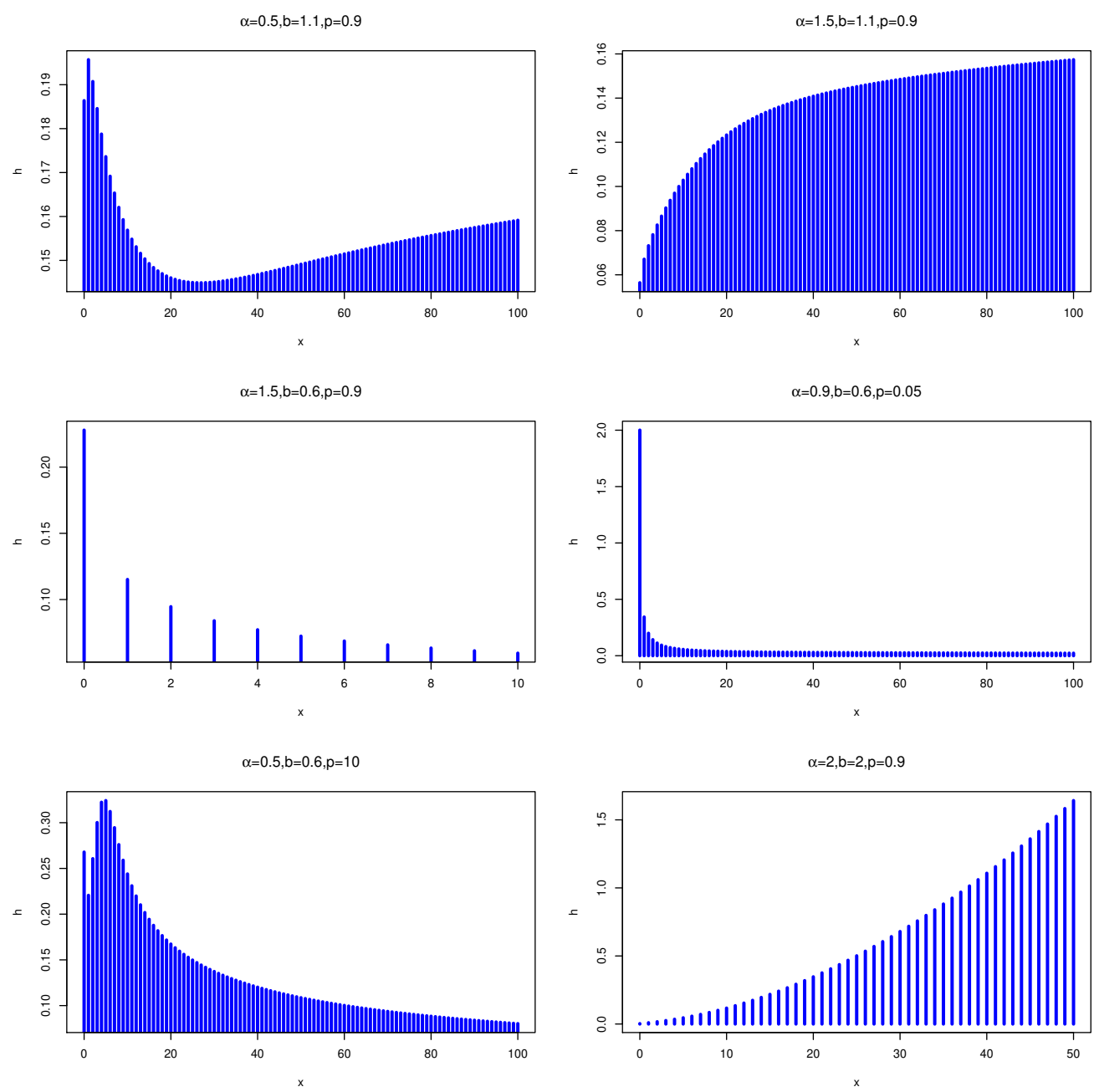

Figure 2. hrfs of the $\operatorname{DWMOE}(\alpha, b, \rho)$ distribution for some parameter values.

\subsection{General Characteristics of the DWMOE Distribution}

In this section, we study some general properties of the DWMOE distribution.

2.1.1. Quantiles, Probability Generating Function, Mean and Variance

Suppose $X$ follows the DWMOE with $\operatorname{cdf} G(x)=1-\bar{G}(x)$. The quantile function $X=Q(u), 0<u<1$ of DWMOE is given as

$$
X=\left\{\frac{1}{\log \rho}\left[\log \left(\frac{\exp \left\{-\left[\log \left(\frac{1}{1-u}\right)\right]^{\frac{1}{b}}\right\}}{\alpha+\bar{\alpha} \exp \left\{-\left[\log \left(\frac{1}{1-u}\right)\right]^{\frac{1}{b}}\right\}}\right)\right]\right\}-1,
$$

where $u$ follows uniform distribution with support $(0,1)$. Equation (7) can be used to simulate the DWMOE random variable. First, simulate a random variable $u$ and compute the value of $X$ in (7), which is not necessarily an integer. The DWMOE random variate $X$ is the largest integer $\leq X$, denoted as $[X]$. 
In particular, the Median $M$ is given as

$$
M=\left\{\frac{1}{\log \rho}\left[\log \left(\frac{\exp \left\{-[\log (2)]^{\frac{1}{b}}\right\}}{\alpha+\bar{\alpha} \exp \left\{-[\log (2)]^{\frac{1}{b}}\right\}}\right)\right]\right\}-1
$$
by

The probability generating function (pgf) of the $\operatorname{DWMOE}(\alpha, b, \rho)$ distribution is given

$$
P(s)=1+(s-1) \sum_{x=1}^{\infty} s^{x-1} \exp \left(-\left\{-\log \left[\frac{\alpha \rho^{x}}{1-\bar{\alpha} \rho^{x}}\right]\right\}^{b}\right)
$$

The mean and variance do not have compact forms, but still the solutions of following equation gives mean and variance of random variable having $\operatorname{DWMOE}(\alpha, b, \rho)$ model.

$$
E(X)=\sum_{x=1}^{\infty} \exp \left(-\left\{-\log \left[\frac{\alpha \rho^{x}}{1-\bar{\alpha} \rho^{x}}\right]\right\}^{b}\right)
$$

and

$$
V(X)=\sum_{x=1}^{\infty}(2 x-1) \exp \left(-\left\{-\log \left[\frac{\alpha \rho^{x}}{1-\bar{\alpha} \rho^{x}}\right]\right\}^{b}\right)-\left[\sum_{x=1}^{\infty} \exp \left(-\left\{-\log \left[\frac{\alpha \rho^{x}}{1-\bar{\alpha} \rho^{x}}\right]\right\}^{b}\right)\right]^{2}
$$

Using statistical software, the mean and variance of the $\operatorname{DWMOE}(\alpha, b, \rho)$ distribution for different values of $\alpha, b$ and $\rho$ are calculated in Table 1 . From this, we can say that the mean increases with $\rho$ and $\alpha$ for different values of $b$. Moreover, depending on the values of

\begin{tabular}{|c|c|c|c|c|}
\hline & $\begin{array}{l}\rho \longrightarrow \\
\alpha \\
\downarrow\end{array}$ & 0.2 & 0.5 & 0.7 \\
\hline \multirow[b]{2}{*}{$b=0.2$} & 1.2 & $23.8886(6880.8534)$ & $29.2683(11,009.7047)$ & $36.9219(14,310.5902)$ \\
\hline & 0.9 & $23.8283(6879.0820)$ & $29.1251(11,001.4607)$ & $36.6520(14,291.7716)$ \\
\hline \multirow[b]{2}{*}{$b=1.2$} & 1.2 & $0.2296(0.2294)$ & $1.0254(1.4259)$ & $2.4205(5.4258)$ \\
\hline & 0.9 & $0.1680(0.1741)$ & $0.8224(1.1803)$ & $2.0072(4.5549)$ \\
\hline \multirow[b]{2}{*}{$b=2$} & 1.2 & $0.1166(0.1032)$ & $0.9268(0.5908)$ & $2.2734(2.0174)$ \\
\hline & 0.9 & $0.0566(0.0534)$ & $0.6978(0.4811)$ & $1.8268(1.6304)$ \\
\hline \multirow[b]{2}{*}{$b=5$} & 1.2 & $0.0011(0.0011)$ & $0.9671(0.1231)$ & $2.3879(0.4845)$ \\
\hline & 0.9 & $8.5316 \times 10^{-7}\left(8.5316 \times 10^{-7}\right)$ & $0.7933(0.1662)$ & $1.9016(0.3986)$ \\
\hline & 1.2 & $1.1009 \times 10^{-20}\left(1.1009 \times 10^{-20}\right)$ & $0.9934(0.0067)$ & $2.5263(0.2673)$ \\
\hline$b=10$ & 0.9 & $1.55 \times 10^{-85}\left(1.55 \times 10^{-85}\right)$ & $0.9471(0.0500)$ & $1.9548(0.0917)$ \\
\hline
\end{tabular}
$\alpha$ and $\rho$, the mean of the distribution can be smaller or greater than its variance. Therefore, the parameters of the $\operatorname{DWMOE}(\alpha, b, \rho)$ distribution can be used to model different data sets.

Table 1. The mean (variance) of $\operatorname{DWMOE}(\alpha, b, \rho)$ for different values of parameters.

\subsubsection{Shannon Entropy}

In probabilistic context, Shannon entropy is a measure of variation of the uncertainty, with higher entropy corresponding to less information. For a discrete random variable $X$ with $\mathrm{pmf} g(\mathrm{x})$, the Shannon entropy is defined as

$$
S(x)=-\sum_{x} g(x) \log g(x)
$$




$$
\begin{gathered}
\text { Combining (8) and (5) gives } \\
S(x)=-\sum_{x} \exp \left(-\left\{-\log \left[\frac{\alpha \bar{F}(x)}{1-\bar{\alpha} \bar{F}(x)}\right]\right\}^{b}\right)-\exp \left(-\left\{-\log \left[\frac{\alpha \bar{F}(x+1)}{1-\bar{\alpha} \bar{F}(x+1)}\right]\right\}^{b}\right) \\
\log \exp \left(-\left\{-\log \left[\frac{\alpha \bar{F}(x)}{1-\bar{\alpha} \bar{F}(x)}\right]\right\}^{b}\right)-\exp \left(-\left\{-\log \left[\frac{\alpha \bar{F}(x+1)}{1-\bar{\alpha} \bar{F}(x+1)}\right]\right\}^{b}\right)
\end{gathered}
$$

Consider the another representation of pmf of DWMOE, that is after rearranging the pmf of DWMOE, we get

$$
g(x)=\exp \left(-\left\{-\log \left[\frac{\alpha \rho^{x}}{1-\bar{\alpha} \rho^{x}}\right]\right\}^{b}\right)\left\{1-\left[\exp \left(-\left\{-\log \left[\frac{\alpha \rho^{x+1}}{1-\bar{\alpha} \rho^{x+1}}\right]\right\}^{b}-\left(-\left\{-\log \left[\frac{\alpha \rho^{x}}{1-\bar{\alpha} \rho^{x}}\right]\right\}^{b}\right)\right)\right]\right\}
$$

Note that when $b \rightarrow \infty$ and $0<\alpha<1$ then $S(x) \rightarrow 0$. This indicates that smaller values of $b$ increase the uncertainty in the distribution.

\section{Estimation Methods}

To acquire the unknown model parameters, we will look at two estimation methods in this section. Maximum likelihood estimation (MLE) and the Bayesian estimation method are the two methods.

\subsection{MLE Method}

Let $X=\left(X_{1}, \ldots, X_{n}\right)$ a random sample of size $n$ from the DWMOE distribution and $x=\left(x_{1}, \ldots, x_{n}\right)$ its observed values. The log-likelihood equation of the vector $\Omega=(\alpha, b, \rho)$ are given by

$$
l(\Omega)=\sum_{i=1}^{n} \log \left[\exp \left(-\left\{-\log \left[\frac{\alpha \rho^{x_{i}}}{1-\bar{\alpha} \rho^{x_{i}}}\right]\right\}^{b}\right)-\exp \left(-\left\{-\log \left[\frac{\alpha \rho^{\left(x_{i}+1\right)}}{1-\bar{\alpha} \rho^{\left(x_{i}+1\right)}}\right]\right\}^{b}\right)\right] .
$$

The non-linear likelihood equations are obtained by differentiating Equation (10) with respect to the parameters $\alpha, b$, and $\rho$, respectively:

$$
\begin{aligned}
& \frac{\partial l(\Omega)}{\partial \alpha}=\frac{b}{\alpha} \sum_{i=1}^{n} \frac{\frac{1-\rho^{x_{i}}}{1-\bar{\alpha} \rho^{x_{i}}}\left\{-\log \left[\frac{\alpha \rho^{x_{i}}}{1-\bar{\alpha} \rho^{x_{i}}}\right]\right\}^{b-1} \exp \left(-\left\{-\log \left[\frac{\alpha \rho^{x_{i}}}{1-\bar{\alpha} \rho^{x_{i}}}\right]\right\}^{b}\right)}{\exp \left(-\left\{-\log \left[\frac{\alpha \rho^{x_{i}}}{1-\bar{\alpha} \rho^{x_{i}}}\right]\right\}^{b}\right)-\exp \left(-\left\{-\log \left[\frac{\alpha \rho^{\left(x_{i}+1\right)}}{\left.\left.\left.1-\bar{\alpha} \rho^{\left(x_{i}+1\right)}\right]\right\}^{b}\right)}\right.\right.\right.} \\
& -\frac{b}{\alpha} \sum_{i=1}^{n} \frac{\frac{1-\rho^{\left(x_{i}+1\right)}}{1-\bar{\alpha} \rho^{\left(x_{i}+1\right)}}\left\{-\log \left[\frac{\alpha \rho^{\left(x_{i}+1\right)}}{1-\bar{\alpha} \rho \rho^{\left(x_{i}+1\right)}}\right]\right\}^{b-1} \exp \left(-\left\{-\log \left[\frac{\alpha \rho^{\left(x_{i}+1\right)}}{1-\bar{\alpha} \rho^{\left(x_{i}+1\right)}}\right]\right\}^{b}\right)}{\exp \left(-\left\{-\log \left[\frac{\alpha \rho^{x_{i}}}{1-\bar{\alpha} \rho^{x_{i}}}\right]\right\}^{b}\right)-\exp \left(-\left\{-\log \left[\frac{\alpha \rho^{\left(x_{i}+1\right)}}{1-\bar{\alpha} \rho^{\left(x_{i}+1\right)}}\right]\right\}^{b}\right)},
\end{aligned}
$$

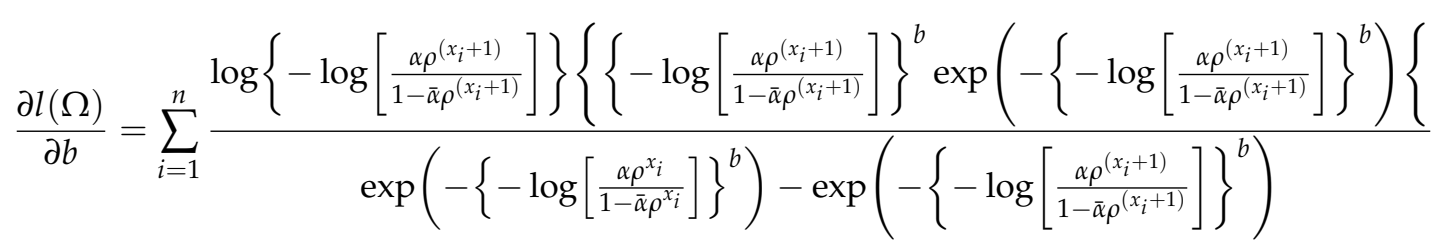

$$
\begin{aligned}
& -\sum_{i=1}^{n} \frac{\log \left\{-\log \left[\frac{\alpha \rho^{x_{i}}}{1-\bar{\alpha} \rho^{x_{i}}}\right]\right\}\left\{-\log \left[\frac{\alpha \rho^{x_{i}}}{1-\bar{\alpha} \rho^{x_{i}}}\right]\right\}^{b} \exp \left(-\left\{-\log \left[\frac{\alpha \rho^{x_{i}}}{1-\bar{\alpha} \rho^{x_{i}}}\right]\right\}^{b}\right)}{\exp \left(-\left\{-\log \left[\frac{\alpha \rho^{x_{i}}}{1-\bar{\alpha} \rho^{x_{i}}}\right]\right\}^{b}\right)-\exp \left(-\left\{-\log \left[\frac{\alpha \rho^{\left(x_{i}+1\right)}}{1-\bar{\alpha} \rho \rho^{\left(x_{i}+1\right)}}\right]\right\}^{b}\right)},
\end{aligned}
$$


and

$$
\begin{aligned}
& \frac{\partial l(\Omega)}{\partial \alpha}=\frac{b}{\rho} \sum_{i=1}^{n} \frac{\frac{x_{i}}{1-\bar{\alpha} \rho^{x_{i}}}\left\{-\log \left[\frac{\alpha \rho^{x_{i}}}{1-\bar{\alpha} \rho^{x_{i}}}\right]\right\}^{b-1} \exp \left(-\left\{-\log \left[\frac{\alpha \rho^{x_{i}}}{1-\bar{\alpha} \rho^{x_{i}}}\right]\right\}^{b}\right)}{\exp \left(-\left\{-\log \left[\frac{\alpha \rho^{x_{i}}}{1-\bar{\alpha} \rho^{x_{i}}}\right]\right\}^{b}\right)-\exp \left(-\left\{-\log \left[\frac{\alpha \rho^{\left(x_{i}+1\right)}}{1-\bar{\alpha} \rho^{\left(x_{i}+1\right)}}\right]\right\}^{b}\right)} \\
& -\frac{b}{\rho} \sum_{i=1}^{n} \frac{\frac{x_{i}+1}{1-\bar{\alpha} \rho^{\left(x_{i}+1\right)}}\left\{-\log \left[\frac{\alpha \rho^{\left(x_{i}+1\right)}}{1-\bar{\alpha} \rho \rho^{\left(x_{i}+1\right)}}\right]\right\}^{b-1} \exp \left(-\left\{-\log \left[\frac{\alpha \rho^{\left(x_{i}+1\right)}}{1-\bar{\alpha} \rho\left(x_{i}+1\right)}\right]\right\}^{b}\right)}{\exp \left(-\left\{-\log \left[\frac{\alpha \rho^{x_{i}}}{1-\bar{\alpha} \rho^{x_{i}}}\right]\right\}^{b}\right)-\exp \left(-\left\{-\log \left[\frac{\alpha \rho^{\left(x_{i}+1\right)}}{1-\bar{\alpha} \rho^{\left(x_{i}+1\right)}}\right]\right\}^{b}\right)},
\end{aligned}
$$

Equating (11)-(13) with zeros and solving simultaneously by using iterative methods such as Newton-Raphson, we obtain the MLEs of $\alpha, b$, and $\rho$. The asymptotic inference for the parameter vector $\xi$ can be based on the normal approximation of the MLE of $\xi$. Indeed, under some regular conditions stated in [30], we have $\hat{\xi}$ is approximately normally distributed with mean $\xi$ and asymptotic variance-covariance matrix $\Sigma_{\xi}$.

\subsection{Bayesian Estimation Model}

We explore the Square error type of loss function in Bayesian estimation. Several authors discussed this loss function based on various models [31,32]. Moreover, several authors discussed Bayesian estimation for parameters of the discrete distribution. For example, see [33-35], etc. For the Bayesian analysis, the prior distributions for each parameter of DWMOE distribution are essential. When prior information about the parameters is lacking, the Bayesian study might use the non-informative prior. Most previous studies of Weibull and Marshall-Olkin family distributions have Gamma prior distribution. Therefore, we supposed the informative priors for parameters $\alpha$ and $b$. The independent gamma distribution is our prior distribution of choice since it plays a significant role in estimating the parameters. While the parameter $\rho$, we used Jeffreys' rule.

The independent joint prior density function of $\alpha, b$ can be written as follows:

$$
\pi(\alpha, b) \propto \alpha^{s_{1}-1} b^{s_{2}-1} e^{-\left(h_{1} \alpha+h_{2} b\right)}
$$

The prior distribution of $\rho$ may be considered to be

$$
\pi(\rho) \propto \frac{1}{\sqrt{\rho(1-\rho)}}
$$

The joint prior density function of $\Omega$ can be written as follows:

$$
\pi(\Omega) \propto \frac{\alpha^{s_{1}-1} b^{s_{2}-1}}{\sqrt{\rho(1-\rho)}} e^{-\left(h_{1} \alpha+h_{2} b\right)}
$$

From the likelihood function and joint prior function (16), the joint posterior density function of $\Omega$ is obtained. The joint posterior of the DWMOE distribution can be written as

$$
\Pi(\Omega \mid x) \propto \frac{\alpha^{s_{1}-1} b^{s_{2}-1}}{\sqrt{\rho(1-\rho)}} e^{-\left(h_{1} \alpha+h_{2} b\right)} \sum_{i=1}^{n}\left[\exp \left(-\left\{-\log \left[\frac{\alpha \rho^{x_{i}}}{1-\bar{\alpha} \rho^{x_{i}}}\right]\right\}^{b}\right)-\exp \left(-\left\{-\log \left[\frac{\alpha \rho^{\left(x_{i}+1\right)}}{1-\bar{\alpha} \rho^{\left(x_{i}+1\right)}}\right]\right\}^{b}\right)\right],
$$


Using the most common loss function (symmetric), which is a squared error. Bayesian estimators of $\tilde{\Omega}$ based on the squared error loss function are defined by Markov Chain Monte Carlo (MCMC).

$$
\begin{aligned}
S(\tilde{\Omega})= & E(\tilde{\Omega}-\Omega)^{2} \\
& \int_{0}^{\infty} \int_{0}^{\infty} \int_{0}^{\infty}(\tilde{\Omega}-\Omega)^{2} \Pi(\Omega \mid x) d \alpha d p d \rho .
\end{aligned}
$$

As the integrals given by (18) cannot be estimated directly. Because of this, we used the MCMC to find an approximate value of integrals. A significant sub-class of the MCMC techniques is the Gibbs sampling and more general Metropolis within Gibbs samplers. The Metropolis-Hastings (MH) bound with the Gibbs sampling were the two most common instances of the MCMC method. Because of the similarity of the MH algorithm with acceptance-rejection sampling, each iteration of the algorithm produces a corresponding candidate value from the proposal distribution. We used the $\mathrm{MH}$ within the Gibbs sampling steps to produce random samples of conditional posterior densities from the DWMOE distribution family:

$$
\begin{aligned}
& \Pi(\alpha \mid p, \rho, x) \propto \alpha^{s_{1}-1} e^{-h_{1} \alpha} \sum_{i=1}^{n}\left[\exp \left(-\left\{-\log \left[\frac{\alpha \rho^{x_{i}}}{1-\bar{\alpha} \rho^{x_{i}}}\right]\right\}^{b}\right)-\exp \left(-\left\{-\log \left[\frac{\alpha \rho^{\left(x_{i}+1\right)}}{1-\bar{\alpha} \rho^{\left(x_{i}+1\right)}}\right]\right\}^{b}\right)\right], \\
& \Pi(b \mid \alpha, \rho, x) \propto b^{s_{2}-1} e^{-h_{2} b} \sum_{i=1}^{n}\left[\exp \left(-\left\{-\log \left[\frac{\alpha \rho^{x_{i}}}{1-\bar{\alpha} \rho^{x_{i}}}\right]\right\}^{b}\right)-\exp \left(-\left\{-\log \left[\frac{\alpha \rho^{\left(x_{i}+1\right)}}{1-\bar{\alpha} \rho^{\left(x_{i}+1\right)}}\right]\right\}^{b}\right)\right],
\end{aligned}
$$

and

$$
\Pi(\rho \mid \alpha, b, x) \propto \frac{1}{\sqrt{\rho(1-\rho)}} \sum_{i=1}^{n}\left[\exp \left(-\left\{-\log \left[\frac{\alpha \rho^{x_{i}}}{1-\bar{\alpha} \rho^{x_{i}}}\right]\right\}^{b}\right)-\exp \left(-\left\{-\log \left[\frac{\alpha \rho^{\left(x_{i}+1\right)}}{1-\bar{\alpha} \rho^{\left(x_{i}+1\right)}}\right]\right\}^{b}\right)\right] \text {. }
$$

\section{Confidence Intervals}

In this section, we propose two different methods to construct confidence intervals (CI) for the unknown parameters of the DWMOE distribution, which are asymptotic confidence interval (ACI) in MLE and credible confidence interval in MCMC of $\alpha, b$, and $\rho$.

\subsection{Asymptotic Confidence Intervals}

The most common method to set confidence bounds for the parameters is to use the asymptotic normal distribution of the MLE. In relation to the asymptotic variancecovariance matrix of the MLE of the parameters, Fisher information matrix $I(\Omega)$, where it is composed of the negative second derivatives of the natural logarithm of the likelihood function evaluated at $\hat{\Omega}=(\hat{\alpha}, \hat{b}, \hat{\rho})$. Suppose the asymptotic variance-covariance matrix of the parameter vector $\Omega$ is

$$
I(\hat{\Omega})=\left[\begin{array}{ccc}
I_{\hat{\alpha} \hat{\alpha}} & & \\
I_{\hat{b} \hat{\alpha}} & I_{\hat{b} \hat{b}} & \\
I_{\hat{\rho} \hat{\alpha}} & I_{\hat{\rho} \hat{b}} & I_{\hat{\rho} \hat{\rho}}
\end{array}\right]
$$

where $V(\hat{\Omega})=I^{-1}(\hat{\Omega})$. 
A $100(1-\gamma) \%$ confidence interval for parameter $\Omega$ can be constructed based on the asymptotic normality of the MLE.

$$
\hat{\alpha} \pm Z_{\frac{\gamma}{2}} \sqrt{I_{\hat{\alpha} \hat{\alpha}}}, \hat{b} \pm Z_{\frac{\gamma}{2}} \sqrt{I_{\hat{b} \hat{b}}} \text {, and } \hat{\rho} \pm Z_{\frac{\gamma}{2}} \sqrt{I_{\hat{\rho} \hat{\rho}}}
$$

where $Z_{\frac{\gamma}{2}}$ is the percentile of the standard normal distribution with right tail probability $\frac{\gamma}{2}$.

\subsection{Highest Posterior Density}

The Highest Posterior Density (HPD) Intervals: [36] discussed this technique to generate the HPD intervals of unknown parameters of the benefit distribution. In this study, samples drawn with the proposed $\mathrm{MH}$ algorithm should be used to generate timelapse estimates. From the percentile tail points, for instance, a $1-\gamma \%$ HPD interval can be obtained with two points for $3^{t} h$ parameters of DWMOE distribution from the MCMC sampling outputs. It is sometimes useful to present the posterior median to informally check on possible asymmetry in the posterior density of a parameter.

According to [36], the BCIs of the parameters of DWMOE distribution $\alpha, b, \rho$ can be obtained through the following steps:

1. Arrange $\tilde{\alpha}, \tilde{b}, \tilde{\rho}$ as $\left(\tilde{\alpha}^{[1]} \leq \tilde{\alpha}^{[2]} \leq \cdots \leq \tilde{\alpha}^{[M]}\right),\left(\tilde{b}^{[1]} \leq \tilde{b}^{[2]} \leq \cdots \leq \tilde{b}^{[M]}\right)$ and $\left(\tilde{\rho}^{[1]} \leq\right.$ $\left.\tilde{\rho}^{[2]} \leq \cdots \leq \tilde{\rho}^{[M]}\right)$, where $M$ denotes the length of the generated of MH algorithm.

2. The $100(1-\gamma) \%$ symmetric credible intervals of $\alpha, b, \rho$ are obtained as: $\left(\tilde{\alpha}^{\left[M \frac{\gamma}{2}\right]}, \tilde{\alpha}^{\left[M\left(1-\frac{\gamma}{2}\right)\right]}\right)$,

$$
\left(\tilde{b}^{\left[M \frac{\gamma}{2}\right]}, \tilde{b}^{\left[M\left(1-\frac{\gamma}{2}\right)\right]}\right) \text { and }\left(\tilde{\rho}^{\left[M \frac{\gamma}{2}\right]}, \tilde{\rho}^{\left[M\left(1-\frac{\gamma}{2}\right)\right]}\right) \text {. }
$$

\section{Simulation}

In order to compare the classical estimation methods, the Monte-Carlo simulation procedure is carried out: MLE, and Bayesian estimation method under square error loss function based on $\mathrm{MCMC}$, for estimation of DWMOE lifetime distribution parameters by $\mathrm{R}$ program. Monte-Carlo experiments are performed based on $\mathrm{t}$ data generated on 10,000 random DWMOE distribution samples using Equation (7), where $x$ has DWMOE lifetime for various parameter actual values as:

Case $1: \alpha=2 ; b=0.75$ with different $\rho=0.25,0.5,0.85$.

Case 2: $\alpha=2 ; b=2$ with different $\rho=0.25,0.5,0.85$.

Case 3: $\alpha=0.65 ; b=2$ with different $\rho=0.25,0.5,0.85$.

and different sample sizes $n:(25,80$, and 150). We could describe the best methods of estimators as minimizing estimators' Bias, mean squared error (MSE) and confidence interval when $\gamma=0.05$.

The simulation results of the methods presented in this paper for point estimation are summarized in the Tables $2-4$. We consider the Bias, MSE, lower and upper of confidence interval values in order to perform the required comparison between various point estimation methods. The following remarks can be noted from these tables:

1. For fixed actual parameters of DWMOE distribution, the Bias, and MSE decrease as $n$ increases.

2. When $\rho$ increases, the Bias and MSE for all parameters decrease.

3. When $b$ increases, the Bias and MSE for $\alpha$ and $\rho$ parameters decrease.

4. Bayesian estimation is the best estimation method according Bias and MSE.

5. The confidence interval of Bayesian estimation is shorter confidence interval than MLE ACI for parameters of DWMOE distribution. 
Table 2. Bias, MSE, Lower, and Upper of CIs for parameters of WMOE distribution: Case 1 with different value of $\rho$

\begin{tabular}{|c|c|c|c|c|c|c|c|c|c|c|}
\hline \multirow[b]{2}{*}{$\rho$} & \multirow[b]{2}{*}{$\mathbf{n}$} & & \multicolumn{4}{|c|}{ MLE } & \multicolumn{4}{|c|}{ Bayesian } \\
\hline & & & Bias & MSE & Lower & Upper & Bias & MSE & Lower & Upper \\
\hline \multirow{9}{*}{0.25} & \multirow{3}{*}{25} & $\alpha$ & 0.2495 & 0.4825 & 0.9782 & 3.5208 & 0.1108 & 0.1755 & 1.4369 & 2.9437 \\
\hline & & $b$ & 0.3604 & 0.1941 & 0.6137 & 1.6072 & 0.1306 & 0.0272 & 0.6855 & 1.0716 \\
\hline & & $\rho$ & 0.1488 & 0.0276 & 0.2538 & 0.5439 & 0.1339 & 0.0219 & 0.2683 & 0.5074 \\
\hline & \multirow{3}{*}{80} & $\alpha$ & 0.0522 & 0.1849 & 1.2152 & 2.8893 & 0.0239 & 0.0273 & 1.7041 & 2.3423 \\
\hline & & $b$ & 0.2732 & 0.0978 & 0.7251 & 1.3213 & 0.1167 & 0.0215 & 0.6969 & 1.0366 \\
\hline & & $\rho$ & 0.1414 & 0.0241 & 0.2664 & 0.5165 & 0.1206 & 0.0163 & 0.3000 & 0.4651 \\
\hline & \multirow{3}{*}{150} & $\alpha$ & 0.1958 & 0.1536 & 1.5301 & 2.8615 & 0.0231 & 0.0201 & 1.7841 & 2.3271 \\
\hline & & $b$ & 0.2462 & 0.0711 & 0.7956 & 1.1967 & 0.1311 & 0.0203 & 0.7421 & 1.0302 \\
\hline & & $\rho$ & 0.1380 & 0.0206 & 0.3115 & 0.4645 & 0.1222 & 0.0160 & 0.3077 & 0.4321 \\
\hline \multirow{9}{*}{0.5} & \multirow{3}{*}{25} & $\alpha$ & -0.0402 & 0.2096 & 1.0459 & 2.8738 & 0.1275 & 0.0408 & 1.9028 & 2.3497 \\
\hline & & $b$ & 0.3161 & 0.1707 & 0.5328 & 1.5994 & 0.1194 & 0.0183 & 0.7461 & 0.9896 \\
\hline & & $\rho$ & 0.0624 & 0.0112 & 0.3906 & 0.7341 & 0.0723 & 0.0060 & 0.4238 & 0.6467 \\
\hline & \multirow{3}{*}{80} & $\alpha$ & -0.0144 & 0.1235 & 1.1361 & 2.7351 & 0.0249 & 0.0241 & 1.7244 & 2.3009 \\
\hline & & $b$ & 0.2003 & 0.0527 & 0.7306 & 1.1700 & 0.0906 & 0.0144 & 0.7017 & 1.0009 \\
\hline & & $\rho$ & 0.0592 & 0.0091 & 0.4885 & 0.6957 & 0.0645 & 0.0058 & 0.5175 & 0.6249 \\
\hline & \multirow{3}{*}{150} & $\alpha$ & 0.0237 & 0.0337 & 1.6843 & 2.3894 & 0.0240 & 0.0224 & 1.7417 & 2.3112 \\
\hline & & $b$ & 0.1786 & 0.0375 & 0.7814 & 1.0758 & 0.0856 & 0.0135 & 0.6822 & 0.9720 \\
\hline & & $\rho$ & 0.0838 & 0.0079 & 0.5270 & 0.6406 & 0.0230 & 0.0048 & 0.4848 & 0.6360 \\
\hline \multirow{9}{*}{0.85} & \multirow{3}{*}{25} & $\alpha$ & -0.0706 & 0.1226 & 1.2567 & 2.6021 & 0.0794 & 0.0646 & 1.6848 & 2.5829 \\
\hline & & $b$ & 0.1413 & 0.0406 & 0.6096 & 1.1730 & 0.0397 & 0.0072 & 0.6569 & 0.9542 \\
\hline & & $\rho$ & 0.0102 & 0.0011 & 0.7993 & 0.9211 & -0.0101 & 0.0010 & 0.8169 & 0.8953 \\
\hline & \multirow{3}{*}{80} & $\alpha$ & -0.0949 & 0.0749 & 1.4013 & 2.4090 & 0.0527 & 0.0627 & 1.5694 & 2.5802 \\
\hline & & $b$ & 0.0963 & 0.0146 & 0.7028 & 0.9897 & 0.0547 & 0.0067 & 0.6901 & 0.9238 \\
\hline & & $\rho$ & 0.0142 & 0.0005 & 0.8298 & 0.8987 & 0.0042 & 0.0003 & 0.8225 & 0.8907 \\
\hline & \multirow{3}{*}{150} & $\alpha$ & -0.0538 & 0.0261 & 1.6475 & 2.2450 & 0.0080 & 0.0238 & 1.7425 & 2.3330 \\
\hline & & $b$ & 0.0871 & 0.0105 & 0.7311 & 0.9431 & 0.0542 & 0.0058 & 0.6958 & 0.9046 \\
\hline & & $\rho$ & 0.0127 & 0.0003 & 0.8411 & 0.8844 & 0.0067 & 0.0002 & 0.8301 & 0.8810 \\
\hline
\end{tabular}


Table 3. Bias, MSE, Lower, and Upper of CIs for parameters of WMOE distribution: Case 2 with different value of $\rho$

\begin{tabular}{|c|c|c|c|c|c|c|c|c|c|c|}
\hline \multirow[b]{2}{*}{$\rho$} & \multirow[b]{2}{*}{$\mathbf{n}$} & & \multicolumn{4}{|c|}{ MLE } & \multicolumn{4}{|c|}{ Bayesian } \\
\hline & & & Bias & MSE & Lower & Upper & Bias & MSE & Lower & Upper \\
\hline \multirow{9}{*}{0.25} & \multirow{3}{*}{25} & $\alpha$ & 0.3139 & 0.1366 & 1.9312 & 2.6966 & 0.1365 & 0.0751 & 1.6867 & 2.5906 \\
\hline & & $b$ & 0.9532 & 1.2137 & 1.8699 & 4.0364 & 0.1257 & 0.0248 & 1.9302 & 2.3134 \\
\hline & & $\rho$ & 0.1161 & 0.0145 & 0.3031 & 0.4292 & 0.1026 & 0.0141 & 0.2932 & 0.4037 \\
\hline & \multirow{3}{*}{80} & $\alpha$ & 0.2935 & 0.0954 & 2.1048 & 2.4821 & 0.0493 & 0.0250 & 1.7261 & 2.3206 \\
\hline & & $b$ & 0.9093 & 0.9120 & 2.3368 & 3.4818 & 0.0948 & 0.0209 & 1.8694 & 2.2937 \\
\hline & & $\rho$ & 0.1204 & 0.0141 & 0.3432 & 0.3976 & 0.1123 & 0.0138 & 0.3418 & 0.4161 \\
\hline & \multirow{3}{*}{150} & $\alpha$ & 0.2742 & 0.0787 & 2.1572 & 2.3912 & 0.0505 & 0.0175 & 1.7838 & 2.2669 \\
\hline & & $b$ & 0.9100 & 0.8728 & 2.4952 & 3.3248 & 0.0762 & 0.0088 & 1.9658 & 2.1777 \\
\hline & & $\rho$ & 0.1122 & 0.0135 & 0.3051 & 0.3993 & 0.1027 & 0.0124 & 0.3483 & 0.4075 \\
\hline \multirow{9}{*}{0.5} & \multirow{3}{*}{25} & $\alpha$ & 0.1388 & 0.0416 & 1.8460 & 2.4317 & 0.1110 & 0.0407 & .8659 & 4055 \\
\hline & & $b$ & 0.5284 & 0.4035 & 1.8371 & 3.2198 & 0.0590 & 0.0070 & 1.9559 & 2.1861 \\
\hline & & $\rho$ & 0.0600 & 0.0042 & 0.5116 & 0.6084 & 0.0591 & 0.0040 & 0.5232 & 0.6014 \\
\hline & \multirow{3}{*}{80} & $\alpha$ & 0.1404 & 0.0261 & 1.9833 & 2.2974 & 0.0362 & 0.0241 & 1.7758 & 729 \\
\hline & & $b$ & 0.5210 & 0.3119 & 6 & 2.9 & 0 & 0.0 & 2.1868 & 2.2654 \\
\hline & & $\rho$ & 0.0627 & 0.0041 & 58 & 0.58 & 0.0624 & 0.0040 & 0.5327 & 0.5860 \\
\hline & \multirow{3}{*}{150} & $\alpha$ & 0.1070 & 0.0124 & 1.7 & 2.2510 & 0.0246 & 0.0121 & 2.0471 & 2.1669 \\
\hline & & $b$ & 0.3693 & 0.1465 & 1.9060 & 2.2538 & 0.0589 & 0.0058 & 2.1972 & 2.2566 \\
\hline & & $\rho$ & 0.0626 & 0.0040 & 0.5367 & 0.5915 & 0.0630 & 0.0039 & 0.5440 & 0.5812 \\
\hline \multirow{9}{*}{0.85} & \multirow{3}{*}{25} & $\alpha$ & 0.0117 & 0.0028 & 1.9101 & 2.1133 & 0.0095 & 0.0021 & 1.9548 & 2.1258 \\
\hline & & $b$ & 0.0584 & 0.0217 & 1.7930 & 2.3238 & 0.0159 & 0.0055 & 1.8639 & 2.1513 \\
\hline & & $\rho$ & 0.0048 & 0.00013 & 0.8341 & 0.8755 & 0.0043 & 0.00012 & 0.8279 & 0.8804 \\
\hline & \multirow{3}{*}{80} & $\alpha$ & 0.0242 & 0.0023 & 1.9423 & 2.1061 & 0.0038 & 0.0020 & 1.9735 & 2.2802 \\
\hline & & $b$ & 0.0563 & 0.0208 & 1.8565 & 2.3562 & 0.0225 & 0.0019 & 1.8279 & 2.2248 \\
\hline & & $\rho$ & 0.0061 & 0.00008 & 0.8439 & 0.8683 & 0.0060 & 0.00007 & 0.8421 & 0.8707 \\
\hline & \multirow{3}{*}{150} & $\alpha$ & 0.0082 & 0.00015 & 1.9908 & 2.0256 & 0.0036 & 0.00010 & 1.9978 & 2.0210 \\
\hline & & $b$ & 0.0324 & 0.0020 & 1.9708 & 2.0940 & 0.0211 & 0.0018 & 1.9855 & 2.0919 \\
\hline & & $\rho$ & 0.0062 & 0.00006 & 0.8482 & 0.8642 & 0.0061 & 0.00005 & 0.8456 & 0.8675 \\
\hline
\end{tabular}


Table 4. Bias, MSE, Lower, and Upper of CIs for parameters of WMOE distribution: Case 3 with different value of $\rho$

\begin{tabular}{|c|c|c|c|c|c|c|c|c|c|c|}
\hline \multirow[b]{2}{*}{$\rho$} & \multirow[b]{2}{*}{$\mathbf{n}$} & & \multicolumn{4}{|c|}{ MLE } & \multicolumn{4}{|c|}{ Bayesian } \\
\hline & & & Bias & MSE & Lower & Upper & Bias & MSE & Lower & Upper \\
\hline \multirow{9}{*}{0.25} & \multirow{3}{*}{25} & $\alpha$ & 0.6215 & 0.4291 & 0.8654 & 1.6776 & 0.2448 & 0.1164 & 0.5450 & 1.3491 \\
\hline & & $b$ & 0.8058 & 0.7366 & 2.2257 & 3.3858 & 0.0258 & 0.0304 & 1.9406 & 2.1294 \\
\hline & & $\rho$ & 0.1794 & 0.0761 & 0.2583 & 0.4004 & 0.1764 & 0.0534 & 0.3045 & 0.5271 \\
\hline & \multirow{3}{*}{80} & $\alpha$ & 0.3994 & 0.1825 & 0.7521 & 1.3466 & 0.0977 & 0.0284 & 0.5057 & 1.0224 \\
\hline & & $b$ & 0.4832 & 0.2835 & 2.0445 & 2.9219 & 0.0287 & 0.0138 & 1.8191 & 2.2592 \\
\hline & & $\rho$ & 0.1236 & 0.0157 & 0.3341 & 0.4132 & 0.1087 & 0.0137 & 0.3512 & 0.5246 \\
\hline & \multirow{3}{*}{150} & $\alpha$ & 0.2488 & 0.1497 & 0.6930 & 1.3647 & 0.1178 & 0.0231 & 0.5419 & 0.9682 \\
\hline & & $b$ & 0.3610 & 0.1964 & 2.3010 & 2.9082 & 0.0428 & 0.0118 & 1.8586 & 2.2454 \\
\hline & & $\rho$ & 0.1068 & 0.0118 & 0.3159 & 0.3976 & 0.0836 & 0.0104 & 0.3642 & 0.5011 \\
\hline \multirow{9}{*}{0.5} & \multirow{3}{*}{2} & $\alpha$ & 0.5223 & 0.3338 & 0.6880 & 1.6 & 0.3891 & 32 & 0.5403 & 5798 \\
\hline & & $b$ & 0.7786 & 0.7465 & 2.0440 & 3.5131 & 0.0641 & 0.0702 & 1.9507 & 2.1617 \\
\hline & & $\rho$ & 0.0122 & 0.0030 & 0.4072 & 0.6172 & 0.0453 & 0.0030 & 0.4242 & 0.6474 \\
\hline & \multirow{3}{*}{80} & $\alpha$ & 0.3975 & 0.1886 & 0.7011 & 1.39 & 0.1113 & 0.0291 & 0.4944 & 0.9766 \\
\hline & & $b$ & 0.6600 & 0.4671 & 2.3 & 3.1 & 0. & 0.0147 & 1.8994 & 2.2347 \\
\hline & & $\rho$ & 0.0382 & 0.0029 & 0.4634 & 0.6 & 0.0359 & 0.0028 & 0.5204 & 0.6423 \\
\hline & \multirow{3}{*}{150} & $\alpha$ & 0.3428 & 0.1849 & 0.8647 & 1.2903 & 0.1206 & 0.0261 & 0.5750 & 0.9880 \\
\hline & & $b$ & 0.6597 & 0.4553 & 2.3812 & 2.9382 & 0.0490 & 0.0121 & 1.9415 & 2.3053 \\
\hline & & $\rho$ & 0.0289 & 0.0014 & 0.4814 & 0.5764 & 0.0218 & 0.0013 & 0.5306 & 0.6450 \\
\hline \multirow{9}{*}{0.85} & \multirow{3}{*}{25} & $\alpha$ & 0.1351 & 0.0477 & 0.4457 & 1.1245 & 0.1219 & 0.0411 & 0.5329 & 1.3685 \\
\hline & & $b$ & 0.2723 & 0.1236 & 1.8326 & 2.7121 & 0.0746 & 0.0151 & 1.9471 & 2.3112 \\
\hline & & $\rho$ & 0.0021 & 0.0004 & 0.8105 & 0.8938 & -0.0046 & 0.0004 & 0.8183 & 0.8853 \\
\hline & \multirow{3}{*}{80} & $\alpha$ & 0.1386 & 0.0343 & 0.5382 & 1.0390 & 0.0405 & 0.0122 & 0.4971 & 0.9237 \\
\hline & & $b$ & 0.2066 & 0.0756 & 1.8366 & 2.5767 & 0.0504 & 0.0103 & 1.8674 & 2.2138 \\
\hline & & $\rho$ & -0.0017 & 0.0002 & 0.8191 & 0.8776 & 0.0016 & 0.0002 & 0.8215 & 0.8798 \\
\hline & \multirow{3}{*}{150} & $\alpha$ & 0.1274 & 0.0212 & 0.6385 & 0.9163 & 0.0591 & 0.0120 & 0.5335 & 0.8672 \\
\hline & & $b$ & 0.1926 & 0.0727 & 2.0980 & 2.4304 & 0.0208 & 0.0056 & 1.9020 & 2.1739 \\
\hline & & $\rho$ & 0.0065 & 0.0002 & 0.7832 & 0.8299 & 0.0041 & 0.0002 & 0.7858 & 0.8482 \\
\hline
\end{tabular}

\section{Applications}

In this section, we illustrate the usefulness of the newly proposed DWMOE distribution. We fit the DWMOE distribution to two data sets and compare the results with those of the fitted Kumaraswamy-geometric (KG), discrete Lindley (DL), and geometric models. For comparing the these distributions, we estimated the values of unknown parameters by the maximum likelihood method with standard errors (SE). Moreover, the fitted distributions are compared using the $-\log$ likelihood (- $\log \mathrm{L}$ ), AIC (Akaike Information Criterion), BIC (Bayesian Information Criterion), Kolmogorov-Smirnov (K-S) statistic, the corresponding $p$-values, the values of Anderson-Darling $\left(A^{*}\right)$ and Cramér von Mises $\left(W^{*}\right)$. The considered data sets are presented below. 
Data set 1 : The first dataset represents COVID-19 data belong to Australia of 32 days, which are recorded from 3 September to 4 October 2020, see, ref. [37]. The data set is:

615591159811796760885752352812374223

Data set 2: The second data set refers the integer part of the lifetime of fifty devices in weeks is given by [38]. The data set is:

0011111236711121818181818213236404546475055 6063636767676772757982828384848485858585858686

Tables 5 and 6 represent the values of descriptive study for the fitted DWMOE, KG, DL and geometric models for Data set 1 and Data set 2, respectively. From the study, the smallest $-\log \mathrm{L}, \mathrm{AIC}, \mathrm{BIC}, \mathrm{K}-\mathrm{S}$ statistic, $A^{*}, W^{*}$ and the highest $p$-values are obtained for the DWMOE distribution. From these results, the new DWMOE distribution is the adequate model than others. Therefore, it can be used for fitting these data sets. Figures 3 and 4 give the estimated pdfs for Data set 1 and Data set 2, respectively. These plots also indeed affirm that DWMOE distribution is clearly a competitive model for considered datasets. Thus, the new model may be a best alternative to the considered discrete models for modeling these real data sets.

Table 5. Estimated values, $-\log \mathrm{L}, \mathrm{AIC}, \mathrm{BIC}, \mathrm{K}-\mathrm{S}$ statistics, $p$-value, $A^{*}$ and $W^{*}$ for data set 1 .

\begin{tabular}{|c|c|c|c|c|c|c|c|c|}
\hline Distribution & Estimates(SE) & $-\log L$ & AIC & BIC & K-S & $p$-Value & $A^{*}$ & $W^{*}$ \\
\hline DWMOE & $\begin{array}{c}\hat{\alpha}=1657.1914 \\
(4233.4787) \\
\hat{b}=0.421(0.1050) \\
\hat{\rho}=0.3446(0.5445)\end{array}$ & 91.11431 & 188.2286 & 192.6258 & 0.1209 & 0.737 & 0.54066 & 0.0831 \\
\hline KG & $\begin{array}{l}\hat{a}=3.1744(0.0171) \\
\hat{b}=0.2943(0.0526) \\
\hat{p}=0.5717(0.0062)\end{array}$ & 94.9914 & 195.9828 & 200.38 & 0.19379 & 0.1807 & 1.89 & 0.2395 \\
\hline DL & $\hat{a}=3.1744(0.0171)$ & 97.12205 & 196.2441 & 197.7098 & 0.1620 & 0.3703 & 1.3817 & 0.1894 \\
\hline geometric & $\hat{a}=0.1194(0.0198)$ & 98.01666 & 198.0333 & 199.499 & 0.25464 & 0.03153 & 1.3817 & 0.1894 \\
\hline
\end{tabular}

Table 6. Estimated values, $-\log \mathrm{L}, \mathrm{AIC}, \mathrm{BIC}, \mathrm{K}-\mathrm{S}$ statistics, $p$-value, $A^{*}$ and $W^{*}$ for data set 2 .

\begin{tabular}{|c|c|c|c|c|c|c|c|c|}
\hline Distribution & Estimates(SE) & $-\log L$ & AIC & BIC & K-S & $p$-Value & $A^{*}$ & $W^{*}$ \\
\hline DWMOE & $\begin{array}{c}\hat{\alpha}=11.5621(12.5867) \\
\hat{b}=0.6771(0.1510) \\
\hat{\rho}=0.9488(0.0138)\end{array}$ & 238.0759 & 482.1519 & 487.8879 & 0.17892 & 0.0814 & 2.364 & 0.3069 \\
\hline KG & $\begin{array}{l}\hat{a}=0.4987(0.3082) \\
\hat{b}=0.1129(0.1299) \\
\hat{p}=0.8367(0.1758)\end{array}$ & 240.1928 & 486.3855 & 492.1216 & 0.1855 & 0.0641 & 2.876 & 0.4761 \\
\hline DL & $\hat{a}=0.0424(0.0042)$ & 249.9767 & 501.9534 & 503.8654 & 0.20147 & 0.0345 & 6.2977 & 0.6529 \\
\hline geometric & $\hat{p}=0.02142(0.0030)$ & 241.6264 & 485.2527 & 487.1647 & 0.19310 & 0.048 & 0.1931 & 3.0769 \\
\hline
\end{tabular}



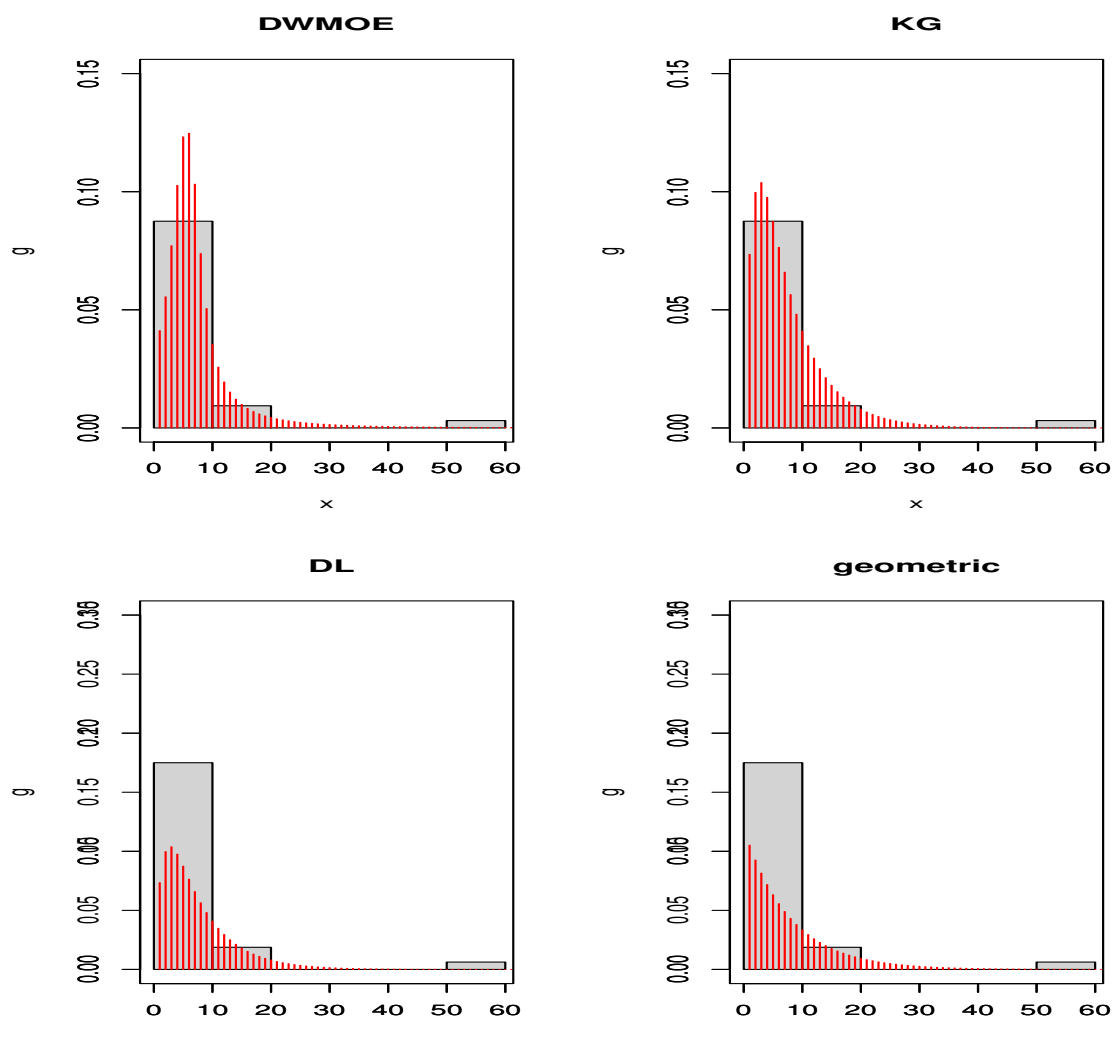

Figure 3. Plots of estimated pdfs of distributions for Data set 1 .
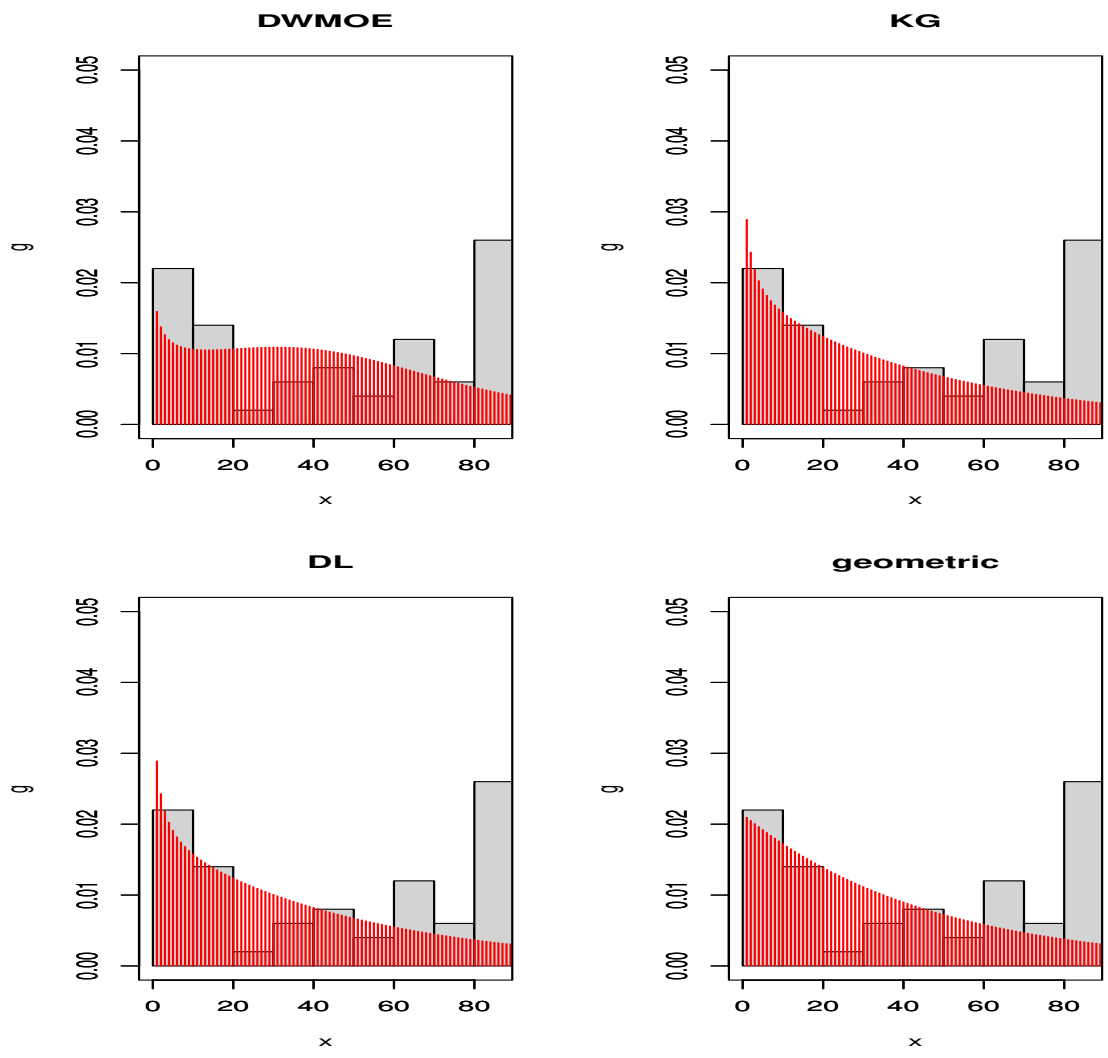

Figure 4. Plots of estimated pdfs of distributions for Data set 2. 


\section{Conclusions}

In this paper, we proposed a new discrete Weibull Marshall-Olkin family of distributions with two shape parameters. A special case of this family, discrete Weibull Marshall-Olkin exponential (DWMOE) distribution is studied in detail. The model parameters have been estimated by the maximum likelihood estimation and Bayesian methods. Simulation study is carried out to assess the performance of the different estimation methods. Finally, two real data sets are analyzed to show the importance and flexibility of specified distribution. We hope that the proposed model will serve as a substitute to various discrete distributions exist in Statistical literature.

Author Contributions: Conceptualization, J.G.; Data curation, E.M.A.; Funding acquisition, O.S.B.; Investigation, J.J.; Methodology, J.G.; Project administration, O.S.B.; Resources, J.J.; Software, F.J.; Validation, J.J.; Writing—original draft, J.G.; Writing—review \& editing, R.A.K.S. All authors have read and agreed to the published version of the manuscript.

Funding: This manuscript is supported by Digiteknologian TKI-ymparisto project A74338(ERDF, Regional Council of Pohjois-Savo).

Institutional Review Board Statement: Not applicable.

Informed Consent Statement: Not applicable.

Data Availability Statement: Not applicable.

Conflicts of Interest: The authors declare no conflict of interest.

\section{References}

1. Marshall, A.W.; Olkin, I. A new method for adding a parameter to a family of distributions with application to the exponential and Weibull families. Biometrica 1997, 84, 641-652. [CrossRef]

2. Castellares, F.; Lemonte, A.J. On the Marshall-Olkin extended distributions. Commun. Stat.-Theory Methods 2016, 45, 4537-4555. [CrossRef]

3. Cordeiro, G.M.; Lemonte, A.J.; Ortega, E.M.M. The Marshall-Olkin family of distributions: mathematical properties and new models. J. Stat. Theory Pract. 2014, 8, 343-366. [CrossRef]

4. Economou, P.; Caroni, C. Parametric proportional odds frailty models. Commun. Stat.-Simul. Comput. 2007, 36, 579-592. [CrossRef]

5. Lam, K.F.; Leung, T.L. Marginal likelihood estimation for proportional odds models with right censored data. Lifetime Data Anal. 2001, 7, 39-54. [CrossRef] [PubMed]

6. Nanda, A.K.; Das, S. Stochastic orders of the Marshall-Olkin extended distribution. Stat. Probab. Lett. 2012, 82, 295-302. [CrossRef]

7. Rao, G.S.; Ghitany, M.E.; Kantam, R.R.L. Reliability test plans for Marshall-Olkin extended exponential distribution. Appl. Math. Sci. 2009, 55, 2745-2755.

8. Rubio, F.J.; Steel, M.F.J. On the Marshall-Olkin transformation as a skewing mechanism. Comput. Stat. Data Anal. 2012, 56, 2251-2257. [CrossRef]

9. Tahir, M.H.; Nadarajah, S. Parameter induction in continuous univariate distributions:Well established G families. Ann. Braz. Acad. Sci. 2015, 87, 539-568. [CrossRef]

10. Jayakumar, K. and Thomas, M. On a generalization of Marshall-Olkin scheme and its application to Burr type XII distribution. Stat. Pap. 2008, 49, 421-439. [CrossRef]

11. Aly, E.; Benkherouf, E. A new family of distributions based on probability generating functions. Sankhya B-Appl. Interdiscip. Stat. 2011, 73, 70-80. [CrossRef]

12. Nadarajah, S.; Jayakumar, K.; Ristić, M.M. A new family of lifetime models. J. Stat. Comput. Simul. 2012, 83, 1-16. [CrossRef]

13. Alizadeh, M.; Cordeiro, G.; Brito, E.; Demétrio, C. The beta Marshall-Olkin family of distributions. J. Stat. Distrib. Appl. 2015, 23, 546-557. [CrossRef]

14. Alizadeh, M.; Tahir, H.M.; Cordeiro, G.M.; Zubair, M.; Hamedani, G.G. The Kumaraswamy Marshal-Olkin family of distributions. J. Egypt. Math. Soc. 2015, 23, 546-557. [CrossRef]

15. Sankaran, K.K.; Jayakumar, K. A new extended uniform distribution. International J. Stat. Distrib. Appl. 2016, 2, 35-41. [CrossRef]

16. Yousof, H.M.; Afify, A.; Nadarajah, S.; Hamedani, G.; Aryal, G. The Marshall-Olkin Generalized-G Family of Distributions with Applications. Statistica 2018, 78, 273-295.

17. Jayakumar, K.; Sankaran, K.K. Discrete Linnik Weibull distribution. Commun. Stat.-Simul. Comput. 2019, 48, 3092-3117. [CrossRef]

18. Korkmaz, M.C.; Cordeiro, G.M.; Yousof, H.M.; Pescim, R.R.; Afify, A.Z.; Nadarajah, S. The Weibull Marshall-Olkin family: regression model and applications to censored data. Commun. Stat.-Theory Methods 2019, 48, 4171-4194. [CrossRef]

19. Alzaatreh, A.; Lee, C.; Famoye, F. A new method for generating families of continuous distributions. Metron 2013, 71, 63-79. [CrossRef] 
20. Chakraborty, S.; Chakravarty, D. A new discrete probability distribution with integer support on $(-\infty, \infty)$. Commun. Stat.-Theory Methods 2016, 45, 492-505. [CrossRef]

21. Krishna, H.; Pundir, P.S. Discrete Burr and discrete Pareto distributions. Stat. Methodol. 2009, 6, 177-188. [CrossRef]

22. Lisman, J.H.C.; van Zuylen, M.C.A. Note on the generation of the most probable frequency distribution. Stat. Neerl. 1972, 26, 19-23. [CrossRef]

23. Nakagawa, T.; Osaki, S. The discrete Weibull distribution. IEEE Trans. Reliab. 1975, 24, 300-301. [CrossRef]

24. Sato, H.; Ikota, M.; Aritoshi, S.; Masuda, H. A new defect distribution meteorology with a consistent discrete exponential formula and its applications. IEEE Trans. Semicond. Manuf. 1999, 12, 409-418. [CrossRef]

25. Stein, W.E.; Dattero, R. A new discrete Weibull distribution. IEEE Trans. Reliab. 1984, 33, 196-197. [CrossRef]

26. Jayakumar, K.; Sankaran, K.K. A Discrete Generalization of Marshall-Olkin Scheme and its Application to Geometric Distribution. J. Kerala Stat. Assoc. 2017, 28, 1-21.

27. Jayakumar, K.; Sankaran, K.K. A generalization of discrete Weibull distribution. Commun. Stat.-Simul. Comput. 2017, 47, 6064-6078. [CrossRef]

28. Sandhya, E.; Prasanth, C.B. Marshall-Olkin discrete uniform distribution. J. Probab. 2014, 2014, 979312. [CrossRef]

29. Chakraborty, S. A new discrete distribution related to generalized gamma distribution and its properties. Commun. Stat.-Theory Methods 2015, 44, 1691-1705. [CrossRef]

30. Cox, D.R.; Hinkley, D.V. Theoretical Statistics; Chapman \& Hall: London, UK, 1974.

31. Bantan, R.; Hassan, A.S.; Almetwally, E.; Elgarhy, M.; Jamal, F.; Chesneau, C.; Elsehetry, M. Bayesian Analysis in Partially Accelerated Life Tests for Weighted Lomax Distribution. CMC-Comput. Mater. Contin. 2021, 68, 2859-2875. [CrossRef]

32. Sabry, M.A.; Almetwally, E.M.; Alamri, O.A.; Yusuf, M.; Almongy, H.M.; Eldeeb, A.S. Inference of fuzzy reliability model for inverse Rayleigh distribution. Aims Math. 2021, 6, 9770-9785. [CrossRef]

33. Archer, E.; Park, I.M.; Pillow, J.W. Bayesian entropy estimation for countable discrete distributions. J. Mach. Learn. Res. 2014, 15, 2833-2868.

34. Ashour, S.K.; Muiftah, M.S.A. Bayesian Estimation of the Parameters of Discrete Weibull Type (I) Distribution. J. Mod. Appl. Stat. Methods 2020, 18, 12. [CrossRef]

35. Irony, T.Z. Bayesian estimation for discrete distributions. J. Appl. Stat. 1992, 19, 533-549. [CrossRef]

36. Chen, M.H.; Shao, Q.M. Monte Carlo estimation of Bayesian credible and HPD intervals, J. Comput. Graph. Stat. 1999, 8, 69-92.

37. Almetwally, E.M.; Ibrahim, G.M. Discrete Alpha Power Inverse Lomax Distribution with Application of COVID-19 Data. Int. J. Appl. Math. Stat. Sci. 2020, 9, 11-22.

38. Aarset, M.V. How to identify a bathtub hazard rate. IEEE Trans. Reliab. 1987, 36, 106-108. [CrossRef] 